\title{
Advances in fiber grating technology for sensor applications
}

\section{Bennion}

I. Bennion, "Advances in fiber grating technology for sensor applications," Proc. SPIE 4185, Fourteenth International Conference on Optical Fiber Sensors, 41855L (9 November 2000); doi: 10.1117/12.2302344

SDIE Event: Fourteenth International Conference on Optical Fiber Sensors, 2000, Venice, Italy 


\title{
Advances in Fiber Grating Technology for Sensor Applications
}

\author{
I Bennion, L Zhang \\ Photonics Research Group, Aston University, Birmingham B4 7ET, UK \\ D A Jackson, D J Webb \\ Applied Optics Group, University of Kent, Canterbury CT2 7NR, UK
}

\begin{abstract}
Sensing techniques employing UV-inscribed fibre Bragg gratings and long period fibre gratings continue to make significant advances. A number of recently established concepts are described offering improved sensor performance, multiplex capacity, and simplicity, and results from new application areas presented.
\end{abstract}

